\title{
Metabolic tumor volume predicts overall survival in patients with primary pulmonary lymphoepithelioma-like carcinoma
}

\author{
CHUN-YU LIN ${ }^{1,2}$, YU-CHUAN CHANG ${ }^{3,4}$, I-TING WANG ${ }^{5}$, MENG-HENG HSIEH ${ }^{1}$, CHIH-WEI WANG $^{6}$, \\ SHU-MIN LIN ${ }^{1}$, CHING-YANG WU ${ }^{7 *}$ and YUEH-FU FANG ${ }^{1 *}$ \\ ${ }^{1}$ Department of Thoracic Medicine, Chang Gung Memorial Hospital at Linkou, Taoyuan 33305; \\ ${ }^{2}$ Department of Pulmonary and Critical Care, Saint Paul's Hospital, Taoyuan 33069; \\ ${ }^{3}$ Department of Medical Imaging and Radiological Sciences, College of Medicine; \\ ${ }^{4}$ Department of Nuclear Medicine and Molecular Imaging Center, Chang Gung Memorial Hospital, Chang Gung University, \\ Taoyuan 33305; ${ }^{5}$ Department of Pulmonary and Critical Care, Mackay Memorial Hospital, Taipei 10491; \\ ${ }^{6}$ Department of Pathology, Chang Gung Memorial Hospital at Linkou; ${ }^{7}$ Department of Surgery, \\ Division of Thoracic and Cardiovascular Surgery, Chang Gung Memorial Hospital at Linkou, \\ Chang Gung University, Taoyuan 33305, Taiwan, R.O.C.
}

Received March 7, 2019; Accepted September 6, 2019

DOI: $10.3892 / 01.2019 .10954$

\begin{abstract}
Pretreatment tumor metabolic burden, measured using fluorine-18 fluorodeoxyglucose positron emission tomography/computerized tomography $\left({ }^{18} \mathrm{~F}-\mathrm{FDG} \mathrm{PET} / \mathrm{CT}\right)$, has been demonstrated to predict outcomes in various types of malignancies. Additionally, Epstein-Barr virus (EBV) serum titer is associated with stages of pulmonary lymphoepithelioma-like carcinoma (LELC). The present study aimed to investigate the prognostic value of the functional parameters of ${ }^{18} \mathrm{~F}-\mathrm{FDG}$ PET/CT in pulmonary LELC and their association with serum EBV DNA. The present retrospective study
\end{abstract}

Correspondence to: Dr Yueh-Fu Fang, Department of Thoracic Medicine, Chang Gung Memorial Hospital at Linkou, 5 Fu-Hsing Street, Kweishan, Taoyuan 33305, Taiwan, R.O.C.

E-mail: dr.fang.yf@gmail.com

Dr Ching-Yang Wu, Department of Surgery, Division of Thoracic and Cardiovascular Surgery, Chang Gung Memorial Hospital at Linkou, Chang Gung University, 5 Fu-Hsing Street, Kweishan, Taoyuan 33305, Taiwan, R.O.C.

E-mail:wu.chingyang@gmail.com

*Contributed equally

Abbreviations: EBV, Epstein-Barr virus: ${ }^{18} \mathrm{~F}-\mathrm{FDG}$ PET/CT, fluorine-18 fluorodeoxyglucose positron emission tomography/computerized tomography; LELC, lymphoepithelioma-like carcinoma; MTV, metabolic tumor volume; NPC, nasopharyngeal carcinoma; $\mathrm{SUV}_{\max }$, maximum standardized uptake value; PFS, progression-free survival; OS, overall survival; TLG, total lesion glycolysis

Key words: prognosis, fluorodeoxyglucose positron emission tomography/computerized tomography, metabolic tumor volume, Epstein-Barr virus DNA, pulmonary lymphoepithelioma-like carcinoma analyzed data from 71 patients with pulmonary LELC; among these, 32 patients with pulmonary LELC underwent pretreatment ${ }^{18}$ F-FDG PET/CT staging between January 2008 and December 2016. EBV viral load and functional parameters of ${ }^{18}$ F-FDG PET/CT were used for survival analysis. Multivariate analysis identified tumor stage IV as a significant predictor of poor progression-free survival [hazard ratio (HR), 4.85; $\mathrm{P}=0.049$ ], whereas elevated total metabolic tumor volume (MTV $\geq 72.6 \mathrm{ml})$ independently predicted worse overall survival (OS; HR, 12.59; $\mathrm{P}=0.024)$. Pretreatment serum EBV DNA titer was significantly positively associated with total $\operatorname{MTV}(\mathrm{P}=0.0337)$ and total lesion glycolysis (TLG; $\mathrm{P}=0.0093)$, but could not predict outcomes. Total MTV was an independent predictor of OS, and may guide clinical management for pulmonary LELC.

\section{Introduction}

Primary pulmonary lymphoepithelioma-like carcinoma (LELC) is a rare lung malignancy $(1,2)$ and patients with primary pulmonary LELC have an improved prognosis compared with patients with other types of lung cancer (2-4); however, few prognostic factors have been described (1).

The utility of fluorine-18 fluorodeoxyglucose positron emission tomography/computerized tomography $\left({ }^{18} \mathrm{~F}-\mathrm{FDG}\right.$ $\mathrm{PET} / \mathrm{CT}$ ) for staging patients with lung cancer has been previously validated (5) and a number of studies have discussed the usage of ${ }^{18} \mathrm{~F}$-FDG PET/CT in pulmonary $\operatorname{LELC}(6,7)$. $\mathrm{Su}$ et al (8) have recently demonstrated that ${ }^{18} \mathrm{~F}-\mathrm{FDG} \mathrm{PET} / \mathrm{CT}$ can more accurately stage cancer and better predict outcomes in patients with pulmonary LELC. Additionally, the functional parameters of ${ }^{18} \mathrm{~F}-\mathrm{FDG} \mathrm{PET} / \mathrm{CT}$, including maximum standardized uptake value $\left(\mathrm{SUV}_{\max }\right)$, metabolic tumor volume (MTV) and total lesion glycolysis (TLG), which represent tumor metabolic activity, entire tumor burden and overall metabolic activity, respectively, are known predictors of 
outcomes in several malignancies, such as breast, rectal cancer and lung cancer (9-12). However, to the best of our knowledge, the prognostic value of these parameters in patients with pulmonary LELC remains unknown.

Similar to that of nasopharyngeal carcinoma (NPC), the etiology of pulmonary LELC is substantially associated with Epstein-Barr virus (EBV) infection in Asian individuals (13), and serum EBV titers in pulmonary LELC are associated with tumor size and stage (14). Furthermore, circulating EBV DNA may be positively associated with tumor burden (15).

Based on the aforementioned results, combined evaluations of imaging parameters and blood biomarkers may lead to the development of improved therapeutic approaches for patients with pulmonary LELC. Therefore, the present study aimed to investigate the prognostic value of the functional parameters of ${ }^{18} \mathrm{~F}$-FDG PET/CT in patients with pulmonary LELC, and to understand the association between these parameters and EBV DNA load.

\section{Materials and methods}

Patients. Between January 2008 and December 2016, 71 individuals with pulmonary LELC were managed at Linkou Medical Center of Chang Gung Memorial Hospital (Taoyuan, Taiwan). This cohort has been previously described $(4,8)$. Pulmonary LELC was diagnosed in all patients based on pathology findings and according to the World Health Organization criteria (16). The present study excluded all patients who had undifferentiated carcinoma without dense lymphoid infiltrates. Routine nasopharyngeal evaluations were performed in all patients to exclude metastatic NPC. Patients with incomplete medical records and those who did not undergo pretreatment ${ }^{18} \mathrm{~F}$-FDG PET/CT evaluation were excluded from the present study. A total of 32 adult patients who underwent pretreatment ${ }^{18} \mathrm{~F}-\mathrm{FDG} \mathrm{PET} / \mathrm{CT}$ for staging were included, comprising 12 men and 20 women with a median age of 60.5 years (range, $48-87$ years). All patients were followed up until June 2017 or until death.

Staging was performed according to the 7th Edition of the American Joint Committee on Cancer guidelines (17). Data on sex, age, smoking status, baseline serum EBV DNA levels, stage, imaging findings and outcomes were obtained. Restaging scans were obtained at 3-month intervals during treatment and were evaluated by a radiologist based on the Response Evaluation Criteria in Solid Tumors, version 1.1 (18). Follow-up information was gathered from patient medical records, and progression-free survival (PFS; defined as time from treatment to time of progression or death) and overall survival (OS; defined as time from treatment to time of death) were calculated. EBV DNA was extracted from plasma samples and amplified using quantitative PCR as described previously (19). Plasma EBV DNA concentration was expressed as the number of copies of EBV genome $/ \mathrm{ml}$ of plasma (20).

Imaging and analysis of ${ }^{18} \mathrm{~F}-\mathrm{FDG} P E T / C T$. All patients were asked to fast for $\geq 4 \mathrm{~h}$ prior to the ${ }^{18} \mathrm{~F}$-FDG PET/CT scan. Blood glucose concentrations were measured prior to positron emission tomography (PET) studies, and the cut-offs were $<120 \mathrm{mg} / \mathrm{dl}$ for non-diabetic patients and $<200 \mathrm{mg} / \mathrm{dl}$ for diabetic patients. Subjects in the resting state were administered intravenous injections of ${ }^{18} \mathrm{~F}-\mathrm{FDG}(5.18 \mathrm{MBq} / \mathrm{kg}$ of body weight), and images were captured at 50 min after tracer administration. Delayed phase images were acquired 90 min after intravenous tracer injection if equivocal lesions were suspected. The MTV and TLG were calculated only for 50-min images. Whole-body PET/computed tomography (CT) emission scans were obtained from the base of the skull to mid-thigh in a Discovery ST16 scanner (GE Healthcare) or a Biograph mCT scanner (Siemens Healthineers). Low-dose CT was used for attenuation correction of PET data and images were reconstructed using CT-based attenuation correction based on an ordered-subset expectation maximization interactive reconstruction algorithm (4 iterations and 10 subsets for the Discovery ST16; 2 iterations and 21 subsets for the Biography mCT). Axial spatial resolution of PET at the center of the gantry, using these reconstruction parameters, was determined to be 4.80 and $2.16 \mathrm{~mm}$ for the Discovery ST16 and Biograph mCT scanners, respectively. The $\mathrm{SUV}_{\max }$ was obtained by drawing regions of interest over the most intense slice of the primary tumor within lesions in the lung and mediastinum. MTV was measured by an SUV-based automated contouring program, which used attenuation-corrected torso ${ }^{18} \mathrm{~F}$-FDG PET images. Boundaries were drawn to include the primary tumor and metastatic sites in axial, coronal and sagittal ${ }^{18} \mathrm{~F}-\mathrm{FDG}$ $\mathrm{PET} / \mathrm{CT}$ images, and voxels within the contouring margin that had an SUV intensity $>2.5$ were incorporated to define the MTV. Total MTV was calculated by adding the MTVs of all malignant lesions in each patient. TLG was calculated by multiplying the MTV of each lesion with the corresponding mean SUV (TLG = mean SUV x MTV) $(21,22)$, and total TLG was calculated by adding the TLGs of all malignant lesions in each patient. Parametric quantification of MTV and TLG was performed using the Syngo MI Workplace software platform, syngoMMWP version VE40A (Siemens Healthineers).

Statistical analysis. Categorical variables were compared using Fisher's exact test and are presented as numbers (percentages) or median values with ranges. The correlation between PET parameters and plasma EBV DNA load was evaluated using Pearson's correlation analysis. Kaplan-Meier survival analysis was used to analyze PFS and OS. Receiver operation characteristic (ROC) curves were used to determine cut-off values for $\mathrm{SUV}_{\max }$, total MTV and TLG. Univariate comparison of survival utilized the log-rank test. All analyses were two-sided. $\mathrm{P}<0.05$ was considered to indicate a statistically significant difference. Statistical analyses were performed using Prism (version 5; GraphPad Software, Inc.) and SPSS (version 20.0; IBM Corp.).

\section{Results}

Patient characteristics. Of the 71 patients with pulmonary LELC, only 32 adult patients who underwent pretreatment ${ }^{18}$ F-FDG PET/CT for staging were included, and their data were analyzed. The demographic characteristics of this cohort of patients, comprising 12 men and 20 women with a median age of 60.5 years (range, 48-87 years), are summarized in Table I. A total of nine patients $(28.1 \%)$ were former or current smokers. Limited to the retrospective nature to this study, 
Table I. Clinical characteristics of patients with pulmonary lymphoepithelioma-like carcinoma.

\begin{tabular}{|c|c|}
\hline Characteristic & $\mathrm{N}(\%)$ \\
\hline Median age, years (range) & $60.5(48-87)$ \\
\hline \multicolumn{2}{|l|}{ Sex } \\
\hline Male & $12(37.5)$ \\
\hline Female & $20(62.5)$ \\
\hline \multicolumn{2}{|l|}{ History of smoking } \\
\hline Former or current smoker & $9(28.1)$ \\
\hline Non-smoker & $23(71.9)$ \\
\hline \multicolumn{2}{|l|}{ ECOG performance status } \\
\hline 0 & $12(37.5)$ \\
\hline 1 & $20(62.5)$ \\
\hline \multicolumn{2}{|l|}{ Stage } \\
\hline I & $10(31.2)$ \\
\hline II & $5(15.6)$ \\
\hline III & $11(34.4)$ \\
\hline IV & $6(18.8)$ \\
\hline EBV DNA level (baseline), copies/ml & \\
\hline Median (range) & $532(66-146,000)$ \\
\hline \multicolumn{2}{|l|}{ Primary treatment } \\
\hline Surgery & $14(43.8)$ \\
\hline Surgery with adjuvant $\mathrm{CT} \pm \mathrm{RT}$ & $4(12.5)$ \\
\hline Neoadjuvant CT \pm RT with surgery & $4(12.5)$ \\
\hline Palliative $\mathrm{CT} \pm \mathrm{RT}$ & $9(28.1)$ \\
\hline RT & $1(3.1)$ \\
\hline
\end{tabular}

CT, chemotherapy; ECOG, Eastern Cooperative Oncology Group; EBV, Epstein-Barr virus; RT, radiotherapy.

the serum EBV DNA level was checked in only nine (28.1\%) patients prior to treatment, all of whom exhibited elevated EBV DNA levels (median, 532 copies/ml; range, 66-146,000 copies/ml).

Associations among tumor stage, EBV DNA load and ${ }^{18}$ F-FDG PET parameters. The cohort comprised 10 (31.3\%) stage I, five (15.6\%) stage II, 11 (34.4\%) stage III and six (18.8\%) stage IV patients with cancer. Tumor stage was significantly associated with total MTV $\left(\mathrm{R}^{2}=0.53 ; \mathrm{P}<0.0001\right)$ and total TLG $\left(\mathrm{R}^{2}=0.40\right.$; $\mathrm{P}=0.0002$, Table II). Pretreatment serum EBV DNA load at baseline, evaluated in nine patients, exhibited a significant positive correlation with total MTV $\left(\mathrm{R}^{2}=0.63 ; \mathrm{P}=0.0337\right)$ and total TLG $\left(\mathrm{R}^{2}=0.77 ; \mathrm{P}=0.0093\right.$, Table II). Data on post-treatment serum EBV DNA load was available for 8 patients, including one patient who succumbed to nosocomial pneumonia after surgery and four who remained alive and disease-free with no detectable serum EBV DNA after therapy.

Survival analysis. During a median follow-up period of 34.1 months (range, 5.1-104.8 months), there were six cases of mortality (five patients succumbed to cancer progression; one patient succumbed to pneumonia and severe sepsis), and tumor progression or recurrence was observed in 11 patients. For
Table II. Correlation analyses of ${ }^{18}$ F-FDG PET/CT functional parameters and tumor stage, EBV DNA levels.

\begin{tabular}{lrrrrr}
\hline & \multicolumn{2}{c}{ Total MTV } & & \multicolumn{2}{c}{ TLG } \\
\cline { 2 - 3 } \cline { 6 - 7 } Parameter & $\mathrm{R}^{2}$ & P-value & & $\mathrm{R}^{2}$ & P-value \\
\hline Stage & 0.53 & $<0.0001$ & & 0.40 & 0.0002 \\
EBV DNA, copies $/ \mathrm{ml}$ & 0.63 & 0.0337 & & 0.77 & 0.0093
\end{tabular}

EBV, Epstein-Barr virus; MTV, metabolic tumor volume; TLG, total lesion glycolysis.

survival analysis, the cut-off values that were selected based on ROC curve analysis for categorization of low and high tumor $\mathrm{SUV}_{\max }$, total MTV, total TLG and serum EBV DNA, were set at 13.7 (area under the curve (AUC), 0.65; $\mathrm{P}=0.298$ ), 72.6 (AUC, 0.9; $\mathrm{P}=0.0056$ ), 278.4 (AUC, 0.9; $\mathrm{P}=0.006$ ) and 236 (AUC, 0.75; $\mathrm{P}=0.4386$ ) copies $/ \mathrm{ml}$, respectively. Univariate analysis demonstrated that high total MTV $(\geq 72.6 \mathrm{ml})$ was associated with poor PFS (hazard ratio (HR), 3.60; 95\% CI, 1.2-37.6; $\mathrm{P}=0.030$; Table III), whereas multivariate analysis identified only stage IV tumors as independent predictors of worse PFS (HR, 4.85; 95\% CI, 1.0-23.3; P=0.049; Table II). Although high EBV DNA load ( $\geq 236$ copies $/ \mathrm{ml}$ ) exhibited a trend toward poorer PFS, this was not identified to be statistically significant (Fig. 1).

Median OS was significantly shorter in patients with stage IV tumors, high total MTV $(\geq 72.6 \mathrm{ml})$ and high total TLG ( $\geq 278.4 \mathrm{~g}$ ) compared with patients with low MTV and low TLG (P<0.05; Fig. 2). Furthermore, only high total MTV $(\geq 72.6 \mathrm{ml}$ ) was a predictor of OS according to multivariate analysis (HR, 12.59; 95\% CI, 1.4-113.7; P=0.024; Table IV).

Two patients with stage IV pulmonary LELC who exhibited divergent values for pretreatment total MTV and total TLG exhibited different outcomes (Fig. 3).

\section{Discussion}

Several functional parameters of ${ }^{18} \mathrm{~F}$-FDG PET, including SUV, MTV and TLG, have been demonstrated to predict outcomes in numerous solid organ tumors, such as breast, colorectal and lung cancer (9-12). To the best of our knowledge, the present study is the first that discusses prognostic values and the association of EBV DNA load and pretreatment ${ }^{18} \mathrm{~F}-\mathrm{FDG}$ PET parameters in primary pulmonary LELC, which is probably due to the rarity of the disease. The present study demonstrated a significant positive association between pretreatment serum EBV DNA load and total MTV or TLG levels. Stage IV tumors were independently associated with poor PFS, and a high total MTV was the only identified predictor of worse OS.

Primary pulmonary LELC is morphologically similar to NPC $(1,2,4)$ and is associated with EBV infection in Asian individuals $(13,14,23)$. Ma et al (24) have demonstrated a positive association between plasma EBV DNA load and MRI-based tumor burden in NPC. Additionally, a study by Chang et al (22) in Taiwan involving 108 patients suggested that tumor TLG, nodal TLG, total TLG and tumor SUV ${ }_{\max }$ 
Table III. Cox proportional hazards model of progression free survival.

\begin{tabular}{|c|c|c|c|c|}
\hline \multirow[b]{2}{*}{ Variable } & \multicolumn{2}{|c|}{ Univariate analysis } & \multicolumn{2}{|c|}{ Multivariate analysis } \\
\hline & $\mathrm{HR}(95 \% \mathrm{CI})$ & P-value & $\mathrm{HR}(95 \% \mathrm{CI})$ & P-value \\
\hline Stage (IV vs. I/II/III) & $5.69(33.6-192.0)$ & $0.002^{\mathrm{a}}$ & $4.85(1.0-23.3)$ & $0.049^{\mathrm{a}}$ \\
\hline EBV DNA, copies/ml ( $\geq 236$ vs. $<236$ ) & $2.79(0.4-19.1)$ & 0.338 & & \\
\hline $\mathrm{SUV}_{\max }, \mathrm{g} / \mathrm{ml}(\geq 13.7$ vs. $<13.7)$ & $4.22(0.7-10.4)$ & 0.136 & & \\
\hline Total MTV, $\mathrm{ml}(\geq 72.6$ vs. $<72.6)$ & $3.60(1.2-37.6)$ & $0.030^{\mathrm{a}}$ & $2.66(0.6-12.8)$ & 0.221 \\
\hline TLG, g ( $\geq 278.4$ vs. $<278.4)$ & $1.81(0.5-7.9)$ & 0.350 & & \\
\hline
\end{tabular}

${ }^{\mathrm{a}} \mathrm{P}<0.05$. HR, hazard ratio; EBV, Epstein-Barr virus; $\mathrm{SUV}_{\max }$, maximum standardized uptake value; MTV, metabolic tumor volume; TLG, total lesion glycolysis.
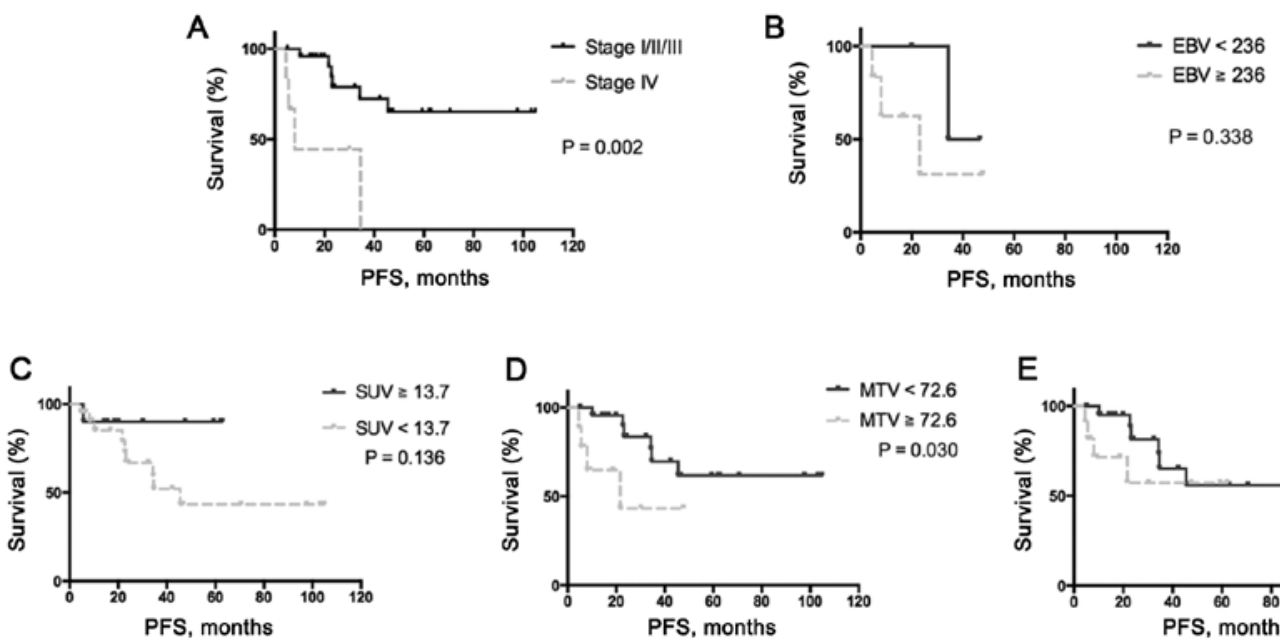

Figure 1. Kaplan-Meier survival analysis of progression-free survival in patients with primary pulmonary lymphoepithelioma-like carcinoma. (A) Tumor stage, (B) serum EBV level, (C) tumor $\mathrm{SUV}_{\max }$, (D) total MTV and (E) total TLG were evaluated. EBV, Epstein-Barr virus; MTV, metabolic tumor volume;

$\mathrm{SUV}_{\max }$, maximum standardized uptake value; TLG, total lesion glycolysis.
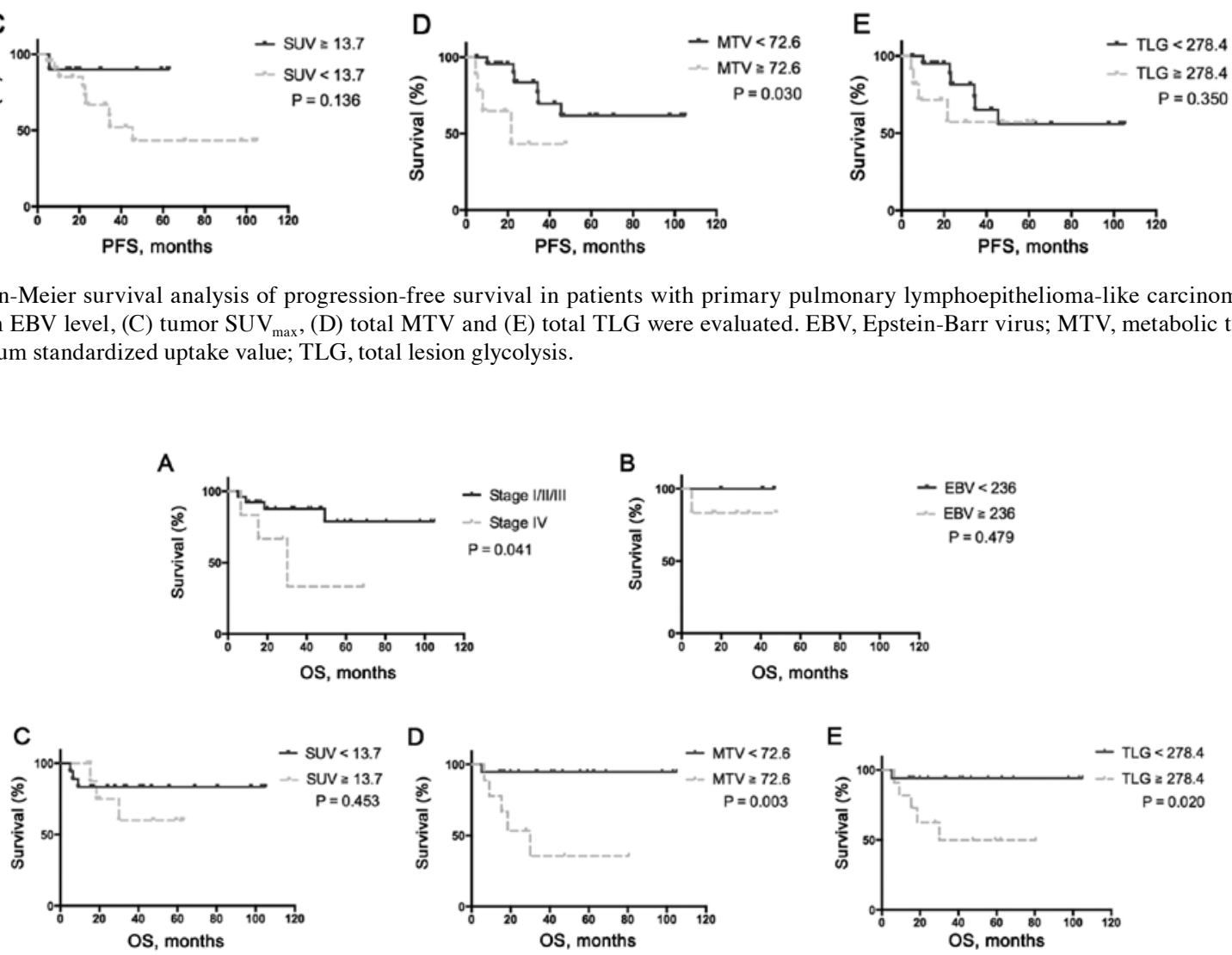

Figure 2. Kaplan-Meier survival analysis of overall survival in patients with primary pulmonary lymphoepithelioma-like carcinoma. (A) Tumor stage, (B) serum EBV level, (C) tumor $\mathrm{SUV}_{\max }$, (D) total MTV and (E) total TLG were evaluated. EBV, Epstein-Barr virus; MTV, metabolic tumor volume; SUV $\mathrm{max}_{\text {max }}$, maximum standardized uptake value; TLG, total lesion glycolysis.

were all significantly associated with plasma EBV DNA load in patients with NPC. However, to the best of our knowledge, the association between EBV viral load and PET parameters in pulmonary LELC has not been previously explored. Although pretreatment serum EBV DNA load was evaluated in only nine patients in the present study, it was significantly positively 
Table IV. Cox proportional hazards model for overall survival.

\begin{tabular}{|c|c|c|c|c|}
\hline \multirow[b]{2}{*}{ Variable } & \multicolumn{2}{|c|}{ Univariate analysis } & \multicolumn{2}{|c|}{ Multivariate analysis } \\
\hline & $\mathrm{HR}(95 \% \mathrm{CI})$ & P-value & HR $(95 \% \mathrm{CI})$ & P-value \\
\hline Stage (IV vs. I/II/III) & $4.17(1.1-68.5)$ & $0.041^{\mathrm{a}}$ & $2.15(0.2-0.9)$ & 0.510 \\
\hline EBV DNA, copies/ml ( $\geq 236$ vs. <236) & $4.48(0.1-286.5)$ & 0.479 & & \\
\hline $\mathrm{SUV}_{\max }, \mathrm{g} / \mathrm{ml}(\geq 13.7$ vs. $<13.7)$ & $1.83(0.4-10.1)$ & 0.453 & & \\
\hline Total MTV, ml ( $\geq 72.6$ vs. <72.6) & $12.60(2.6-90.9)$ & $0.003^{\mathrm{a}}$ & $12.59(1.4-113.7)$ & $0.024^{\mathrm{a}}$ \\
\hline TLG, g ( $\geq 278.4$ vs. <278.4) & $8.40(1.4-37.8)$ & $0.020^{\mathrm{a}}$ & $5.97(0.4-95.6)$ & 0.207 \\
\hline
\end{tabular}

${ }^{\mathrm{a}} \mathrm{P}<0.05$. HR, hazard ratio; EBV, Epstein-Barr virus; $\mathrm{SUV}_{\max }$, maximum standardized uptake value; MTV, metabolic tumor volume; TLG, total lesion glycolysis.
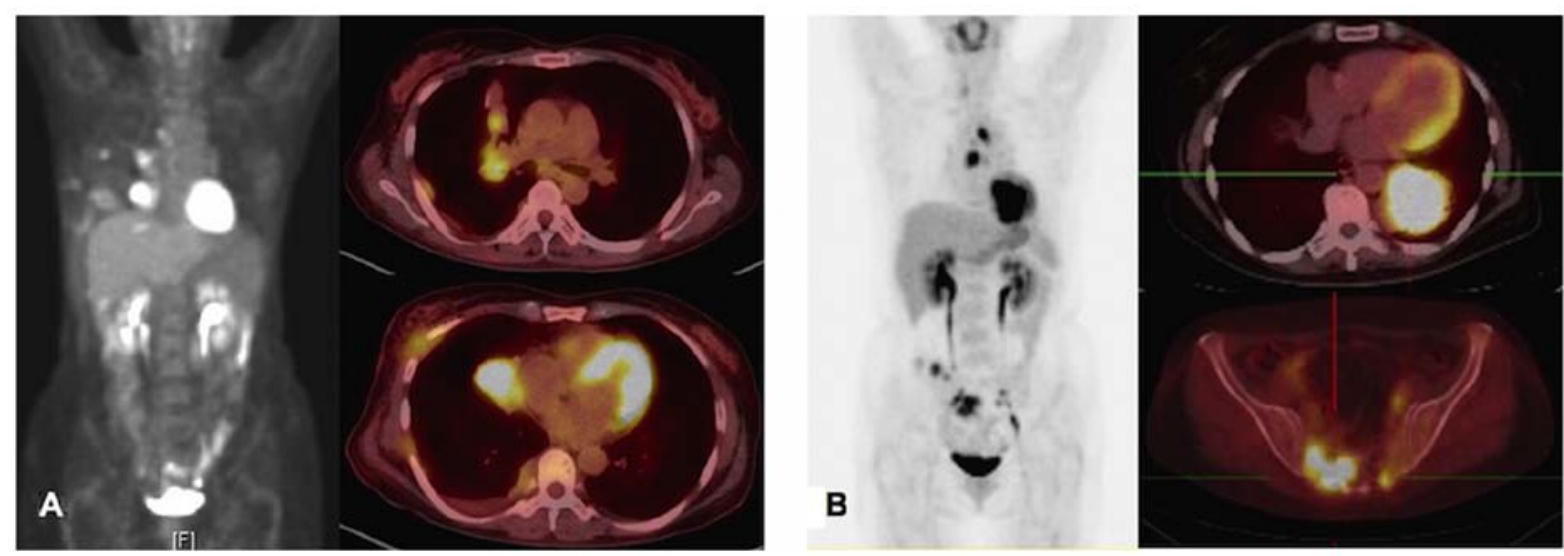

Figure 3. Fluorine-18 fluorodeoxyglucose positron emission tomography/computerized tomography in two patients with stage IV pulmonary lymphoepithelioma-like carcinoma. PFS and OS were worse for the patient with elevated total MTV and TLG. (A) A 61-year-old female with an SUV max $_{\text {ax }}$ of 10.43 , total MTV of $52.76 \mathrm{ml}$ and TLG of $179.82 \mathrm{ml}$. The PFS was 34.6 months and the OS was 68.8 months. (B) A 54-year-old female with an SUV $\mathrm{max}_{\max }$ of 15.46 , total MTV of $197.82 \mathrm{ml}$ and total TLG of $1223.82 \mathrm{ml}$. The PFS was 5.6 months and the OS was 15.4 months. MTV, metabolic tumor volume; PFS, progression-free survival; $\mathrm{SUV}_{\max }$, maximum standardized uptake value; TLG, total lesion glycolysis; OS, overall survival.

associated with total MTV and total TLG, suggesting that the plasma EBV viral load is associated with active tumor burden.

Plasma EBV DNA load in patients with NPC has been demonstrated to have a high prognostic value with respect to long-term survival and distant metastasis in one meta-analysis (25). Chang et al (14) reported that elevated EBV serology titers represent higher tumor stage and larger tumor size in patients with pulmonary LELC. Furthermore, Ngan et al $(15,26)$ demonstrated that the circulating EBV DNA load is associated with treatment response. The results of the present study suggested that EBV DNA load may be positively associated with tumor burden. Data on EBV DNA load before and after treatment was not available for eight patients, and although four patients who had no detectable serum EBV DNA after therapy remained disease-free, the prognostic value of serum EBV DNA could not be evaluated due to the small sample size.

Compared with conventional CT, PET/CT provides morphological and functional information relevant to cancer management (21). Su et al (8) reported that stage and pretreatment ${ }^{18} \mathrm{~F}$-FDG PET were independent prognostic factors of OS in patients with pulmonary LELC; however, that study did not evaluate the prognostic value of functional parameters of
${ }^{18} \mathrm{~F}$-FDG PET. The most commonly used parameter is $\mathrm{SUV}_{\max }$, which represents maximum FDG uptake in a region of interest, and greater FDG uptake by a tumor is associated with worse survival in patients with surgical and non-surgical lung cancer $(27,28)$. Previously, Shen et al (29) have revealed that $\mathrm{SUV}_{\text {max }}$ is a predictor of OS in patients with recurrent NPC. However, since $\mathrm{SUV}_{\max }$ largely depends on tumor size, its prognostic value is questionable (30). Notably, Hoang et al (31) have described contradictory results and suggest that $\mathrm{SUV}_{\max }$ does not exhibit a significant association with survival in patients with advanced non-small cell lung cancer.

Regarding volumetric PET parameters, MTV represents metabolically active tumor volume on PET images (32). Lee et al (33) have demonstrated that MTV has prognostic value for PFS and OS in patients with non-small cell lung cancer who are treated definitively. Additionally, alterations in MTV following treatment are also significantly associated with survival in patients with lung cancer $(34,35)$. Another prognostic PET parameter is TLG, which combines volumetric and metabolic information (21). Chung et al (36) reported that total MTV and TLG are independent prognostic factors of PFS and OS in patients with advanced lung adenocarcinoma in Korea. Another retrospective study by Wang et al (37) 
revealed that high TLG was an independent predictor of poor PFS in patients with epidermal growth factor receptor (EGFR)-mutated advanced lung adenocarcinoma, and of poor PFS and OS in patients with wild-type EGFR carrying lung adenocarcinoma. By contrast, in the patients with pulmonary LELC examined in the present study, neither $\mathrm{SUV}_{\max }$ nor TLG were identified to be associated with PFS or OS. Only stage IV tumors predicted worse PFS. Additionally, the present study demonstrated that only MTV was significantly associated with OS in multivariate analysis. This may be due to TLG being affected by another crucial constituent of TLG, i.e., mean SUV.

There were several limitations in the present study. First, this was a retrospective study, which may have led to a few biases. Pretreatment serum EBV DNA was not routinely evaluated in all patients and posttreatment EBV DNA was not regularly followed up. Second, for the majority of patients, ${ }^{18} \mathrm{~F}$-FDG PET/CT scans were only performed once, and changes of PET parameters could not be evaluated. Additionally, all measurements were from a single center and the sample size was small due to the rarity of the disease. Additionally, the use of MTV and TLG in clinical practice may be premature due to a lack of standardized estimation methodology. A number of different methods and a wide range of threshold levels have been proposed to calculate volume-based PET/CT parameters $(38,39)$. Another factor is the use of two different PET/CT devices [Discovery ST16 scanner (GE Healthcare) and Biograph mCT scanner (Siemens Healthineers)] in the present study, which can affect the measurement of SUV and volumetric PET parameters since SUV measurements can be influenced by a variety of biological factors, including body weight and blood glucose level, and technological factors, including inter-scanner variability and image reconstruction parameters (40). However, to the best of our knowledge, the present study was the first to demonstrate the predictive value of ${ }^{18} \mathrm{~F}$-FDG PET/CT functional parameters in pulmonary LELC, and provide relevant and useful information to clinicians.

Although not considered prognostic, pretreatment serum EBV DNA levels were closely associated with MTV and TLG of ${ }^{18} \mathrm{~F}$-FDG PET/CT in patients with pulmonary LELC. Total MTV was found to be an independent predictor of OS and could be valuable for guiding decision-making during pulmonary LELC management. Further prospective, large-scale studies are warranted to validate the findings of the present study and to evaluate the prognostic values of changes in PET functional parameters following treatment.

\section{Acknowledgements}

Not applicable.

\section{Funding}

YFF and CYW were funded by Chang Gung Medical Research Program Grant (grant no. CMRPG3E1981).

\section{Availability of data and materials}

The datasets used and/or analyzed during the current study are available from the corresponding author on reasonable request.

\section{Authors' contributions}

CYL, ITW, CYW, YCC and YFF conceived and designed the study. ITW, YFF and CYW provided administrative support. CWW, MHH, CYW, YCC and SML provided study materials or patients. CYL, CWW, YFF and YCC collected and assembled data. CYW, CWW, ITW, MHH, YFF and SML analyzed and interpreted the data. YFF and CYW had full access to all data in the present study and take responsibility for the integrity of the data and the accuracy of the data analysis, and they contributed equally to this paper. All authors were involved in writing the manuscript. All authors read and approved the final manuscript.

\section{Ethics approval and consent to participate}

The present study was approved by the Institutional Review Board of Chang Gung Memorial Hospital (approval nos. 201600879B0 and 104-8614C). This was a retrospective study and no modification in the management of patients was required, so the need for informed consent was waived. All personal information was encrypted in the database, and patient data accessed was de-identified. There was no breach of privacy.

\section{Patient consent for publication}

Not applicable.

\section{Competing interests}

The authors declare that they have no competing interests.

\section{References}

1. Liang $\mathrm{Y}$, Wang L, Zhu Y, Lin Y, Liu H, Rao H, Xu G and Rong T: Primary pulmonary lymphoepithelioma-like carcinoma: Fifty-two patients with long-term follow-up. Cancer 118: 4748-4758, 2012

2. He J, Shen J, Pan H, Huang J, Liang W and He J: Pulmonary lymphoepithelioma-like carcinoma: A Surveillance, Epidemiology and end results database analysis. J Thorac Dis 7: 2330-2338, 2015.

3. Lin Z, Situ D, Chang X, Liang W, Zhao M, Cai C, Liu Y and He J: Surgical treatment for primary pulmonary lymphoepithelioma-like carcinoma. Interact Cardiovasc Thorac Surg 23: 41-46, 2016.

4. Lin CY, Chen YJ, Hsieh MH, Wang CW and Fang YF: Advanced primary pulmonary lymphoepithelioma-like carcinoma: Clinical manifestations, treatment, and outcome. J Thorac Dis 9: 123-128, 2017.

5. Lv YL, Yuan DM, Wang K, Miao XH, Qian Q, Wei SZ, Zhu XX and Song Y: Diagnostic performance of integrated positron emission tomography/computed tomography for mediastinal lymph node staging in non-small cell lung cancer: A bivariate systematic review and meta-analysis. J Thorac Oncol 6: 1350-1358, 2011.

6. Chan HY, Tsoi A, Wong MP, Ho JC and Lee EY: Utility of 18F-FDG PET/CT in the assessment of lymphoepithelioma-like carcinoma. Nucl Med Commun 37: 437-445, 2016.

7. Aktas GE, Can N, Demir SS and Sarikaya A: Primary pulmonary lymphoepithelioma-like carcinoma on FDG PET/CT. Nucl Med Mol Imaging 51: 88-92, 2017.

8. Su TP, Ho KC, Wang CW, Lin CY, Liu CY, Yang CT and Yen TC: Prognostic value and clinical impact of pretreatment FDG PET in pulmonary lymphoepithelioma-like carcinoma. Clin Nucl Med 44: e68-e75, 2019.

9. Tateishi U, Gamez C, Dawood S, Yeung HW, Cristofanilli M and Macapinlac HA: Bone metastases in patients with metastatic breast cancer: Morphologic and metabolic monitoring of response to systemic therapy with integrated PET/CT. Radiology 247: 189-196, 2008. 
10. Gu J, Khong PL, Wang S, Chan Q, Law W and Zhang J: Quantitative assessment of diffusion-weighted MR imaging in patients with primary rectal cancer: Correlation with FDG-PET/CT. Mol Imaging Biol 13: 1020-1028, 2011.

11. Chen HH, Chiu NT, Su WC, Guo HR and Lee BF: Prognostic value of whole-body total lesion glycolysis at pretreatment FDG $\mathrm{PET} / \mathrm{CT}$ in non-small cell lung cancer. Radiology 264: 559-566, 2012.

12. Paesmans $M$, Berghmans $T$, Dusart $M$, Garcia $C$, Hossein-Foucher C, Lafitte JJ, Mascaux C, Meert AP, Roelandts M, Scherpereel A, et al: Primary tumor standardized uptake value measured on fluorodeoxyglucose positron emission tomography is of prognostic value for survival in non-small cell lung cancer: Update of a systematic review and meta-analysis by the European Lung Cancer Working Party for the International Association for the Study of Lung Cancer Staging Project. J Thorac Oncol 5: 612-619, 2010.

13. Hayashi T, Haba R, Tanizawa J, Katsuki N, Kadota K, Miyai Y, Bando K, Shibuya S, Nakano M and Kushida Y: Cytopathologic features and differential diagnostic considerations of primary lymphoepithelioma-like carcinoma of the lung. Diagn Cytopathol 40: 820-825, 2012.

14. Chang YL, Wu CT, Shih JY and Lee YC: New aspects in clinicopathologic and oncogene studies of 23 pulmonary lymphoepithelioma-like carcinomas. Am J Surg Pathol 26: 715-723, 2002

15. Ngan RK, Yip TT, Cheng WW, Chan JK, Cho WC, Ma VW, Wan KK, Au SK, Law CK and Lau WH: Circulating Epstein-Barr virus DNA in serum of patients with lymphoepithelioma-like carcinoma of the lung: A potential surrogate marker for monitoring disease. Clin Cancer Res 8: 986-994, 2002.

16. Beasley MB, Brambilla E and Travis WD: The 2004 World Health Organization classification of lung tumors. Semin Roentgenol 40: 90-97, 2005.

17. Goldstraw P: The 7th edition of TNM in lung cancer: What now? J Thorac Oncol 4: 671-673, 2009.

18. Eisenhauer EA, Therasse P, Bogaerts J, Schwartz LH, Sargent D, Ford R, Dancey J, Arbuck S, Gwyther S, Mooney M, et al: New response evaluation criteria in solid tumours: Revised RECIST guideline (version 1.1). Eur J Cancer 45: 228-247, 2009

19. Hung CY, Lin TL, Kuo YC, Hsieh CH, Wang HM and Hsu CL: Progesterone analogues reduce plasma Epstein-Barr virus DNA load and improve pain control in recurrent/metastatic nasopharyngeal carcinoma patients under supportive care. Biomed J 40: 212-218, 2017

20. Hsu CL, Chang KP, Lin CY, Chang HK, Wang CH, Lin TL, Liao CT, Tsang NM, Lee LY, Chan SC, et al: Plasma Epstein-Barr virus DNA concentration and clearance rate as novel prognostic factors for metastatic nasopharyngeal carcinoma. Head Neck 34 : 1064-1070, 2012

21. Khiewvan B, Ziai P, Houshmand S, Salavati A, Ziai P and Alavi A: The role of PET/CT as a prognosticator and outcome predictor in lung cancer. Expert Rev Respir Med 10: 317-330, 2016.

22. Chang KP, Tsang NM, Liao CT, Hsu CL, Chung MJ, Lo CW, Chan SC, Ng SH, Wang HM and Yen TC: Prognostic significance of 18F-FDG PET parameters and plasma Epstein-Barr virus DNA load in patients with nasopharyngeal carcinoma. J Nucl Med 53: 21-28, 2012.

23. Han AJ, Xiong M and Zong YS: Association of Epstein-Barr virus with lymphoepithelioma-like carcinoma of the lung in southern China. Am J Clin Pathol 114: 220-226, 2000.

24. Ma BB, King A, Lo YM, Yau YY, Zee B, Hui EP, Leung SF, Mo F, Kam MK, Ahuja A, et al: Relationship between pretreatment level of plasma Epstein-Barr virus DNA, tumor burden and metabolic activity in advanced nasopharyngeal carcinoma. Int J Radiat Oncol Biol Phys 66: 714-720,2006.

25. Zhang W, Chen Y, Chen L, Guo R, Zhou G, Tang L, Mao Y, Li W, Liu X, Du X, et al: The clinical utility of plasma Epstein-Barr virus DNA assays in nasopharyngeal carcinoma: The dawn of a new era? A systematic review and meta-analysis of 7836 cases. Medicine (Baltimore) 94: e845, 2015.
26. Ngan RK, Yip TT, Cheng WW, Chan JK, Cho WC, Ma VW, Wan KK, Au JS and Law CK: Clinical role of circulating Epstein-Barr virus DNA as a tumor marker in lymphoepithelioma-like carcinoma of the lung. Ann N Y Acad Sci 1022: 263-270, 2004

27. Davies A, Tan C, Paschalides C, Barrington SF, O'Doherty M, Utley $\mathrm{M}$ and Treasure T: FDG-PET maximum standardised uptake value is associated with variation in survival: Analysis of 498 lung cancer patients. Lung Cancer 55: 75-78, 2007.

28. Liu J, Dong M, Sun X, Li W, Xing L and Yu J: Prognostic Value of 18F-FDG PET/CT in surgical non-small cell lung cancer: A meta-analysis. PLoS One 11: e0146195, 2016.

29. Shen T, Tang LQ, Luo DH, Chen QY, Li PJ, Mai DM, Guo SS, Liu LT, Qian CN, Guo X, et al: Different prognostic values of plasma Epstein-Barr virus DNA and maximal standardized uptake value of 18F-FDG PET/CT for nasopharyngeal carcinoma patients with recurrence. PLoS One 10: $0122756,2015$.

30. Soret M, Bacharach SL and Buvat I: Partial-volume effect in PET tumor imaging. J Nucl Med 48: 932-945, 2007.

31. Hoang JK, Hoagland LF, Coleman RE, Coan AD, Herndon JE II and Patz EF Jr: Prognostic value of fluorine-18 fluorodeoxyglucose positron emission tomography imaging in patients with advanced-stage non-small-cell lung carcinoma. J Clin Oncol 26: 1459-1464, 2008

32. Obara $\mathrm{P}$ and $\mathrm{Pu} \mathrm{Y}$ : Prognostic value of metabolic tumor burden in lung cancer. Chin J Cancer Res 25: 615-622, 2013.

33. Lee P, Bazan JG, Lavori PW, Weerasuriya DK, Quon A, Le QT, Wakelee HA, Graves EE and Loo BW: Metabolic tumor volume is an independent prognostic factor in patients treated definitively for non-small-cell lung cancer. Clin Lung Cancer 13: 52-58, 2012.

34. Soussan M, Chouahnia K, Maisonobe JA, Boubaya M, Eder V, Morere JF and Buvat I: Prognostic implications of volume-based measurements on FDG PET/CT in stage III non-small-cell lung cancer after induction chemotherapy. Eur J Nucl Med Mol Imaging 40: 668-676, 2013

35. van Loon J, Offermann C, Ollers M, van Elmpt W, Vegt E, Rahmy A, Dingemans AM, Lambin P and De Ruysscher D: Early CT and FDG-metabolic tumour volume changes show a significant correlation with survival in stage I-III small cell lung cancer: A hypothesis generating study. Radiother Oncol 99: 172-175, 2011.

36. Chung HW, Lee KY, Kim HJ, Kim WS and So Y: FDG PET/CT metabolic tumor volume and total lesion glycolysis predict prognosis in patients with advanced lung adenocarcinoma. J Cancer Res Clin Oncol 140: 89-98, 2014.

37. Wang D, Zhang M, Gao X and Yu L: Prognostic value of baseline 18F-FDG PET/CT functional parameters in patients with advanced lung adenocarcinoma stratified by EGFR mutation status. PLoS One 11: e0158307, 2016.

38. Wang XY, Zhao YF, Liu Y, Yang YK, Zhu Z and Wu N: Comparison of different automated lesion delineation methods for metabolic tumor volume of 18F-FDG PET/CT in patients with stage I lung adenocarcinoma. Medicine (Baltimore) 96: e9365, 2017.

39. Carlier T and Bailly C: State-Of-The-Art and recent advances in quantification for therapeutic Follow-Up in oncology using PET. Front Med (Lausanne) 2: 18, 2015.

40. Adams MC, Turkington TG, Wilson JM and Wong TZ: A systematic review of the factors affecting accuracy of SUV measurements. AJR Am J Roentgenol 195: 310-320, 2010.

This work is licensed under a Creative Commons Attribution-NonCommercial-NoDerivatives 4.0 International (CC BY-NC-ND 4.0) License. 